\title{
A Hybrid Genetic Algorithm for Constrained Combinatorial Problems: An application to promotion planning problems
}

\author{
Paulo A. Pereira, Fernando A. C. C. Fontes, and Dalila B. M. M. Fontes
}

\begin{abstract}
We propose a Hybrid Genetic Algorithm (HGA) for a combinatorial optimization problem, motivated by, and a simplification of, a TV Self-promotion Assignment Problem. The problem consists of, given the weekly self-promotion space (a set of TV breaks with known duration) and a set of products to promote, to assign the products to the breaks in the "best" possible way. The objective is to maximize contacts in the target audience for each product, while satisfying all constraints. The HGA developed incorporates a greedy heuristic to initialize part of the population and uses a repair routine to guarantee feasibility of each member of the population. The HGA works on a simplified version of the problem that, nevertheless, maintains its essential features. The proposed simplified problem is a binary programming problem that has similarities with other known combinatorial optimization problems, such as the assignment problem or the multiple knapsack problem, but has some distinctive features that characterize it as a new problem. Although we were mainly interested in solving problems of high dimension (of about 200 breaks and 50 spots), the quality of the solution was tested on smaller dimension problems for which we could find the exact global minimum using a branch-and-bound algorithm. For these smaller dimension problems we have obtained solutions on average within $1 \%$ of the optimal solution value.
\end{abstract}

keywords Genetic Algorithms, Combinatorial Optimization, TV Self-Promotion Assignment Problem.

Paulo A. Pereira

CMAT and Dept. of Mathematics and Appl., University of Minho, 4800-058 Guimarães, Portugal, ppereira@math.uminho.pt

Fernando A. C. C. Fontes

ISR and Faculdade de Engenharia, Universidade do Porto, 4200-465 Porto, Portugal, faf@ fe.up.pt

Dalila B. M. M. Fontes

LIAAD-INESC Porto L.A. and Faculdade de Economia, Universidade do Porto, 4200-464 Porto, Portugal, fontes@fep.up.pt 


\section{Introduction}

We report on the development of an optimizer based on genetic algorithms which is integrated into a Decision Support System (DSS) to plan the best assignment of the weekly self-promotion space for a TV station. This project was developed for SIC, a commercial TV station that frequently leads audience shares in Portugal. This article concentrates on describing the optimizer which is based on a hybrid GA combining a greedy heuristic and constraint handling based on linear programming relaxations.

Advertising is the main source of income in commercial TV's and advertisement revenues grow with audiences. Therefore TV stations typically reserve a promotion space (broadcasting time) for self-promotion (future programs, etc.). However, the total broadcasting time dedicated to advertisement, both commercial and selfpromotion, is regulated and limited. Hence, the self-promotion time should be kept small, thus optimizing its usage is of upmost importance. The problem consists on, given the weekly self-promotion space (a set of breaks with known duration) and a set of products to promote, assigning the products to the breaks in the "best" possible way. For each product we have a given target audience, and for each break we can estimate how many viewers of each target are watching at the time. Moreover, each product has several assignment constraints to be satisfied. The objective is to maximize contacts in the target audience for each product, while satisfying all constraints.

The weekly problem faced by SIC, typically involves the broadcast of about 1350 spots, chosen from around 90 different ones, in roughly 200 time slots. The duration of each of these time slots, here and hereafter designated by breaks, ranges from 6 to 150 seconds. Given the problem complexity and dimensionality (see [Pereira et al (2008), Pereira (2009)] for a more detailed problem description), only heuristic approaches are expected to be able to obtain solutions in a reasonable amount of time. In the next section, we describe a GA that also incorporates a greedy heuristic to initialize part of the population with "good solutions". While the DSS considers the original problem with all the complexity features, the GA works on a simplified version of the problem that, nevertheless, maintains the essential features of the problem. We have noticed that the simplified problem is a binary programming problem that has similarities with other known combinatorial optimization problems, such as the multiple knapsack problem (see e.g. [Tavares et al (2008), Lin (1998)]).

We propose a hybrid GA approach for the problem. First, we use a greedy heuristic procedure to construct good solutions. Each solution is a spot-break assignment binary matrix. Knowing the maximum and the minimum number of times that each spot can be used, we assign the spot that has the greatest number of times to be used to the break with the larger audience rate for the target of the selected spot. This procedure is repeated until each spot coverage maximum spot insertions have been attained, resulting in a spot-break assignment binary matrix with good coverage. Next, we handle with constraints by using a "repair" procedure to elimate unfeasibilities. There are two type of unfeasible solutions: (i) solutions where the maximum 
time of the break may be exceeded, and (ii) solutions where the minimum number of viewers of a spot may not be satisfied. The population of solutions thus obtained is then evolved using the genetic operators reproduction, crossover, and mutation.

\section{The problem}

The problem can be stated as follows. Let $i=1, \ldots, N_{B}$ be the set of breaks, and $j=1, \ldots, N_{S}$ the set of spots. Each break $i$ and spot $j$ are characterized by their durations $B_{i}$ and $d_{j}$, respectively. Also, let $c_{i j}$ denote the number of viewers that watch spot $j$ when inserted in break $i$. We wish to find a spot-break assignment such that a minimum break usage $b_{i}$ for break $i$ and a minimum number of viewers $C_{j}$, not necessarily different, for spot $j$ are guaranteed. Amongst all possible assignments we are interested on the ones that maximize the number of viewers, from the intended target, watching the spots. This problem can be formulated as given in the following binary programming model.

$$
\begin{array}{ll}
\text { Maximize } & \sum_{i=1}^{N_{B}} \sum_{j=1}^{N_{S}} c_{i j} x_{i j} \\
\text { Subject to: } \sum_{j=1}^{N_{S}} d_{j} x_{i j} \leq & B_{i} \quad \forall 1 \leq i \leq N_{B}, \\
& \sum_{j=1}^{N_{S}} d_{j} x_{i j} \geq b_{i} \quad \forall 1 \leq i \leq N_{B}, \\
& \sum_{i=1}^{N_{B}} c_{i j} x_{i j} \geq C_{j} \quad \forall 1 \leq j \leq N_{S}, \\
& x_{i j} \in\{0,1\} \quad \forall 1 \leq i \leq N_{B} \quad \text { e } \forall 1 \leq j \leq N_{S},
\end{array}
$$

where the decision variables $x_{i j}$ assume the value 1 if spot $j$ is assigned to break $i$ and 0 otherwise.

\section{The hybrid genetic algorithm}

Since Holland first proposed GAs in the early 1970s as computer programs that mimic the evolutionary processes in nature [Holland (1975)], the GAs have been demonstrating their power by successfully being applied to many practical optimization problems in the last decade.

We propose a GA that uses a greedy heuristic followed by a repair procedure to generate a population of feasible solutions. This population is then evolved through reproduction, crossover, and mutation. The reproduction and crossover operators determine which parents will have offspring, and how genetic material is exchanged 
between parents. The stochastic universal sampling (SUS) technique, introduced by [Baker (1987)], is used to select solutions for recombination. Such a selection is proportional to the fitness and exhibits no bias and minimal spread. SUS uses a single random value to sample all of the solutions by choosing them at evenly spaced intervals. Crossover happens between a solution which is located in an even position with the solution located in the adjacent odd position. The single point crossover is used. After selecting one crossover point, two children are obtained by using the binary string from the beginning of the chromosome to the crossover point from one parent, the rest being copied from the other parent. An elitist strategy is used, since some of the best individuals are copied from one generation to the next. This is important since this way it is guaranteed that the best solution is monotonically improving from one generation to the next. However, such a strategy tends to increase the convergence rate. Therefore, and in order to avoid excessive convergence, mutation is also incorporated, through immigration, to allow for randomly introduction of new genetic material.

Greedy heuristic:

In order to generate solutions we have implemented a constructive greedy heuristic procedure that outputs spot-break assignment binary matrices with good coverage.

The solution procedure has basically 3 stages: In stage (i) the show-predefined constraints are converted into spot-constraints. Therefore, except for the break duration constraints which are dealt with differently, we only have spot-constraints. Although some constraints are easily converted, others have required complex procedures to do so. This is the case of the minimum coverage constraints. We have developed an estimator for the number of times each show would need to be advertised since the coverage is a nonlinear and unknown function of the broadcasted spots.

In stage (ii), using the minimum number of viewers $C_{j}$ required for each spot $j$, we estimate the maximum $M b_{j}$ and the minimum $m b_{j}$ number of times that spot $j$ may be used.

Then, in stage (iii) we generate the binary matrix by resorting to an iterative greedy heuristic procedure. Spots are iteratively assigned to breaks, starting with the spot having the highest number of minimum times still to be used (initially $m b_{j}$ ). For such a spot we chose the break with the larger audience rate for the targeted of the selected spot, that still has enough unused time. This is repeated until the coverage $C_{j}$ has been provided for all spots, or the spot has already been used $M b_{j}$ times.

The repair routine:

In addition and in order to guarantee solution feasibility (unfeasible solutions ar mainly due group of constraints that imposes not exceeding the breaks duration) we used a "repair" procedure, inspired in that of Chu and Beasley [Chu (1998)] for multiple knapsack problems. This procedure has two phases: in the first one we guarantee that the break time is not exceeded by removing spots, whenever needed, while in the second phase we try to satisfy spot's number of viewers, or improve the solution, by adding spots to the breaks which have some unused time. 
We start by selecting the most violated break, i.e. with the highest used time above its duration, and then iteratively remove from its spots in ascending order of $u_{i j}$ where

$$
u_{i j}=\frac{c_{i j}}{w_{i} d_{j}}
$$

and $w_{i}$ dual value of the break constraint, obtained by solving a linear programming relaxation of (1). Then, spots with a number of viewers smaller than $C_{j}$ are further inserted into breaks with unused time, in ascending order of time usage benefit. This is obtained as the product of the spot duration by the dual variable of the break constraint $\left(w_{i} d_{j}\right)$. If this not possible swaps with over-satisfied spots are attempted. At the end of this routine we have a feasible solution.

\section{Computational results}

In order to test the proposed GA we have used 60 randomly generated problems, consisting of 5 instances of 12 different sizes. Problem size is represented as $(a, b)$, where $a$ is the number of breaks and $b$ the number of spots. The GA was used 10 times for each of these problems, therefore in the following results each reported figure corresponds to the average value obtained from the 10 runs of the 5 problem instances. In Figure 1 we report on the solution quality and computational time requirements.
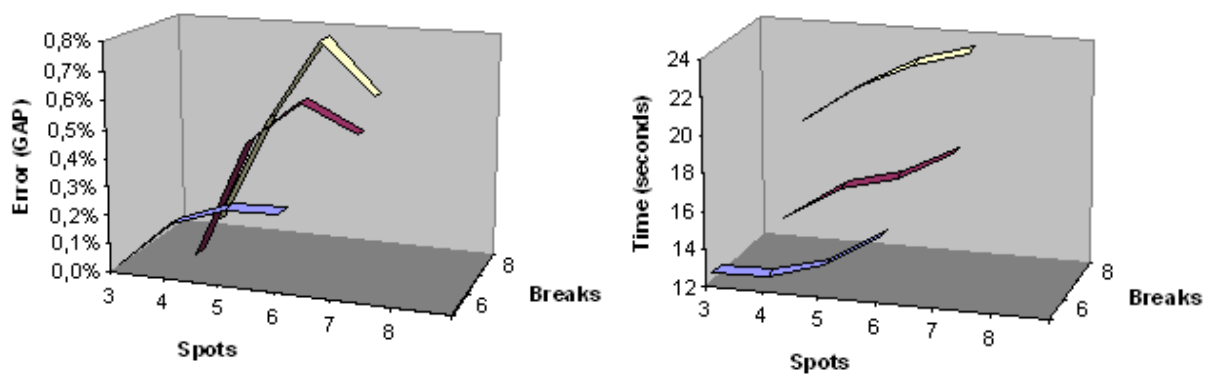

Fig. 1 Computational performance for randomly generated problems.

The time requirements are reported in seconds and the solution quality is the optimality gap obtained as $\frac{G A_{\text {sol }}-O P T}{O P T}$, where $O P T$ is the optimal solution value obtained by solving the problems using a branch-and-bound algorithm.

We have also solved 3 problem instances with 200 breaks and 50 spots, which is the size of the TV station problems. However, for such problems we can only report on computational time requirements, see Table 1, since the branch-and-bound algorithm only solves problems with up to 8 breaks. Again the results reported refer to the average of the 10 runs performed using the GA. 


\begin{tabular}{lcrr}
\hline $\begin{array}{c}\text { Size } \\
(200,50)\end{array}$ & $\begin{array}{c}\text { Best Sol. } \\
\text { Value }\end{array}$ & $\begin{array}{c}\text { Best Sol. } \\
\text { Generation }\end{array}$ & $\begin{array}{c}\text { Best Sol. } \\
\text { Time }\end{array}$ \\
\hline Problem 1 & 107962.30 & 353.35 & 20692.30 \\
Problem 2 & 112203.30 & 398.35 & 20791.30 \\
Problem 3 & 107803.00 & 352.70 & 20782.80 \\
Average & & 368.13 & 20755.50 \\
Std. Dev. & & 21.40 & 44.80 \\
\hline
\end{tabular}

Table 1 Computational performance for real sized problems.

\section{Conclusions}

We consider here a new type of combinatorial optimization problem, motivated by, and a simplification of, the TV Self-promotion Assignment Problem. This problem has similarities with other known combinatorial optimization problems, such as the assignment problem or the multiple knapsack problem, but has some distinctive features that caracterize it as a new problem. A genetic algorithm was developed to address this problem. The algorithm incorporates a greedy heuristic to initialize part of the population and uses a repair routine to guarantee feasibility of each member of the population. Although we were mainly interested in solving problems of high dimension ( 200 breaks by 50 spots), the quality of the solution was tested for smaller dimension problems for which we could find the exact global minimum using a branch-and-bound algorithm. For these smaller dimension problems we have obtained solutions on average within $1 \%$ of the optimal solution value.

\section{References}

[Baker (1987)] Baker J, (1987) Reducing bias and inefficiency in the selection algorithms. In: Proceedings of the Second International Conference on Genetic Algorithms, Lawrence Erlbaum Associates Inc., USA, pp 14-21

[Chu (1998)] Chu J PC e Beasley, (1998) A genetic algorithm for the multidimensional knapsack problem. Journal of Heuristics 4:63-86

[Holland (1975)] Holland J, (1975) Adaptation in Natural and Artificial Systems. University of Michigan Press, Ann Arbor, MI

[Lin (1998)] Lin E, (1998) A biblographical survey on some well-known non-standard knapsack problems. INFOR 36(4):274-317

[Pereira et al (2008)] Pereira P, Fontes F, Fontes D, (2008) A Decision Support System for Planning Promotion Time Slots. In: Operations Research Proceedings 2007: Selected Papers of the Annual International Conference of the German Operations Research Society (GOR), Springer, p 147

[Pereira (2009)] Pereira PA, (2009) Um sistema de apoio à decisão baseado em optimização para o planeamento de auto-promoção de uma estação de televisão. PhD thesis, Departamento de Matemática para a Ciência e Tecnologia, Universidade do Minho, Guimarães, Portugal

[Tavares et al (2008)] Tavares J, Pereira F, Costa E, (2008) Multidimensional knapsack problem: A fitness landscape analysis. IEEE Transactions on Systems, Man, and Cybernetics, Part B 38(3):604-616 